\title{
Plasma proteins take their toll on the joint in osteoarthritis
}

Steven R Goldring*1 and Carla R Scanzello²

\begin{abstract}
In their recent study, Sohn and colleagues identify multiple plasma proteins in the synovial fluid of patients with osteoarthritis $(\mathrm{OA})$ and demonstrate the capacity of several of the proteins to activate macrophages via the innate immune receptor Tolllike receptor-4 (TLR-4). The authors speculate that the plasma proteins transit into the synovial compartment at sites of tissue damage where the endothelial barrier may be compromised, thus bringing them into contact with the articular surface and cells within the synovium. These results demonstrate a novel mechanism by which synovial inflammation can be initiated in patients with $O A$ and how this process may contribute to the pathogenesis of OA joint pathology.
\end{abstract}

The view of osteoarthritis (OA) as strictly a non-inflammatory joint disease has evolved as imaging and histopathological studies have demonstrated that synovial inflammation is present in many patients, and importantly, is associated with increased pain, poorer joint function, and accelerated deterioration of joint structures. In the current article by Sohn and colleagues [1], the authors advance the understanding of how inflammation may be initiated by demonstrating that certain plasma proteins present in OA synovial fluids (SFs) can activate macrophages via the innate immune receptor Toll-like receptor (TLR)-4.

The authors began with a proteomic analysis, utilizing two-dimensional PAGE followed by liquid chromatography-mass spectrometry, to survey proteins in SF from five OA patients. The analysis identified 108 proteins, many of which previously were shown to be increased in OA SF compared to normal, including proteins related to inflammation and immunity and products of extracellular

*Correspondence: goldrings@hss.edu

'Hospital for Special Surgery, Weill Cornell Medical Center, New York, NY, USA

Full list of author information is available at the end of the article matrix degradation and remodeling [2]. Of these, 64 proteins were novel and included histone-related and macrophage-associated proteins, proinflammatory receptors and proteins related to the proinflammatory transcription factor nuclear factor kappa B. As the approach was not optimal for detection of small molecules such as cytokines, they went on to perform multiplex bead-based immunoassays to measure cytokines and chemokines involved in inflammatory processes and compared the levels in normal sera, OA and rheumatoid arthritis sera and OA and rheumatoid arthritis SF. In general, the levels in OA sera samples were only slightly higher than the normal levels and those in the rheumatoid arthritis sera were markedly higher. Some novel mediators were identified by this comparison, including $\mathrm{T}$ cell attracting chemokine (CTAK) and IL-9, which were significantly higher in the OA compared to the normal sera, supporting a role for $\mathrm{T}$ cells in $\mathrm{OA}$ inflammation. Among the OA samples, multiple proinflammatory cytokines and chemokines were much higher in the SF compared to the sera, consistent with overproduction of these cytokines in the joints.

Interestingly, they found that over one-third of proteins identified in the proteomic screen of OA SF were plasma proteins. This is not unexpected, since SF is partially a plasma filtrate, but many of the plasma proteins were increased in OA compared to normal SF. Together with evidence that another plasma protein, fibrinogen, can incite inflammation by stimulating the innate receptor TLR-4, they tested whether other plasma proteins identified in the screen could do the same and demonstrated that three $(\alpha 1 \mathrm{~m}, \alpha 2 \mathrm{~m}$, and Gc-globulin) induced tumor necrosis factor- $\alpha$, IL-1 $\beta$, IL- 6 , and vascular endothelial growth factor release from cultured macrophages. Very recently, other members of this group demonstrated that the complement membrane-attack complex plays a role in the development of inflammation and joint degeneration in an animal model of OA [3]. As plasma can be a source of many proteins, including complement components, the authors speculated that these proteins transit into the synovial compartment at sites of tissue damage where the endothelial barrier may be compromised, thus bringing them into contact with the articular 
surface and cells such as macrophages and synovial fibroblasts.

The authors demonstrated that the effect of these plasma proteins is mediated through TLR-4, as macrophages from mice lacking TLR-4 were unresponsive to the protein treatments. These results are significant for several reasons. First, the authors present data linking SF proteins present in OA joints, including plasma proteins, to activation of inflammatory processes. Second, TLR activation has previously been demonstrated to impact cartilage breakdown $[4,5]$. As macrophages are important mediators of synovial inflammation in OA, the present results extend the potential impact of this pathway to synovitis in OA. Finally, reports of TLR activation by non-microbial products often have been called into question, as low-level endotoxin contamination can often lead to false positive results. This issue was addressed using two different approaches. First, they demonstrated that treatment of the cultures with polymyxin $B$, which binds lipopolysaccharide by charge interactions and prevents it from associating with TLR-4, reverses the lipopolysaccharide response, but does not significantly affect the response to $\alpha 1 \mathrm{~m}, \alpha 2 \mathrm{~m}$, and Gc-globulin. Secondly, they demonstrated that proteinase $\mathrm{K}$ treatment fully or partially reversed the macrophage response to the three plasma proteins, while not affecting lipopolysaccharide.

In conclusion, the article by Sohn and colleagues contributes to our understanding of how TLRs may be activated locally in the setting of OA, by pointing to potential stimuli beyond matrix-derived and stressrelated molecules derived from joint tissues. The observation that plasma proteins present in the OA joint can stimulate macrophages provides important rationale for further study of this pathway in OA models, but also may implicate this pathway in a variety of other local joint conditions that may produce alterations in synovial diffusion properties.
Abbreviations

IL, interleukin; OA, osteoarthritis; SF, synovial fluid; TLR, Toll-like receptor.

\section{Competing interests}

The authors declare that they have no competing interests.

\section{Acknowledgements}

CRS is supported by 1 K08 AR057859, Mentored Clinician Scientist Career Development Award from the National Institute of Arthritis, Musculoskeletal and Skin Diseases.

\section{Author details}

'Hospital for Special Surgery, Weill Cornell Medical Center, New York, NY, USA. ${ }^{2}$ Section of Rheumatology, Departments of Immunology/Microbiology and Biochemistry, Rush University Medical Center, Chicago, IL 60612, USA.

Published: 5 March 2012

\section{References}

1. Sohn DH, Sokolove J, Sharpe O, Erhart JC, Chandra PE, Lahey LJ, Lindstrom TM, Hwang I, Boyer KA, Andriacchi TP, Robinson WH: Plasma proteins present in osteoarthritic synovial fluid can stimulate cytokine production via Toll-like receptor 4. Arthritis Res Ther 2012, 14:R7.

2. Gobezie R, Kho A, Krastins B, Sarracino DA, Thornhill TS, Chase M, Millett PJ, Lee DM: High abundance synovial fluid proteome: distinct profiles in health and osteoarthritis. Arthritis Res Ther 2007, 9:R36.

3. Wang Q, Rozelle AL, Lepus CM, Scanzello CR, Song JJ, Larsen DM, Crish JF, Bebek G, Ritter SY, Lindstrom TM, Hwang I, Wong HH, Punzi L, Encarnacion A, Shamloo M, Goodman SB, Wyss-Coray T, Goldring SR, Banda NK, Thurman JM, Gobezie R, Crow MK, Holers VM, Lee DM, Robinson WH: Identification of a central role for complement in osteoarthritis. Nat Med 2011, 17:1674-1679.

4. Liu-Bryan R, Terkeltaub R: Chondrocyte innate immune myeloid differentiation factor 88-dependent signaling drives procatabolic effects of the endogenous Toll-like receptor 2/Toll-like receptor 4 ligands low molecular weight hyaluronan and high mobility group box chromosomal protein 1 in mice. Arthritis Rheum 2010, 62:2004-2012.

5. Scanzello CR, Plaas A, Crow MK: Innate immune system activation in osteoarthritis: is osteoarthritis a chronic wound? Curr Opin Rheumatol 2008, 20:565-572.

doi:10.1186/ar3741

Cite this article as: Goldring SR, Scanzello CR: Plasma proteins take their toll on the joint in osteoarthritis. Arthritis Research \& Therapy 2012, 14:111. 